\title{
EXTERNALLY POLLUTED WHITE DWARFS WITH DUST DISKS
}

\author{
M. JuRA, ${ }^{1}$ J. FARIHI, ${ }^{2}$ AND B. ZuCKERMAN ${ }^{1}$ \\ Received 2007 February 10; accepted 2007 April 9
}

\begin{abstract}
We report Spitzer Space Telescope photometry of 11 externally polluted white dwarfs. Of the nine stars for which we have IRAC photometry, we find that GD 40, GD 133, and PG 1015+161 each has an infrared excess that can be understood as arising from a flat, opaque, dusty disk. GD 56 also has an infrared excess characteristic of circumstellar dust, but a flat-disk model cannot reproduce the data unless there are grains as warm as $1700 \mathrm{~K}$ and perhaps not even then. Our data support the previous suggestion that the metals in the atmosphere of GD 40 are the result of accretion of a tidally disrupted asteroid with a chondritic composition.
\end{abstract}

Subject headings: circumstellar matter — minor planets, asteroids — white dwarfs

\section{INTRODUCTION}

Gravitational settling of heavy elements is so effective in white dwarfs cooler than $20,000 \mathrm{~K}$ that their atmospheres are expected to be pure hydrogen or pure helium (Paquette et al. 1986), and the $\sim 20 \%$ of these stars that exhibit photospheric metals are thought to be externally polluted (Wolff et al. 2002; Zuckerman et al. 2003; Koester \& Wilken 2006). A promising model to explain these data is that a minor body is perturbed (Debes \& Sigurdsson 2002) to orbit within the tidal radius of the star where it is destroyed and a dusty disk is produced (Jura 2003). Accretion from this disk can explain atmospheric pollutions (Zuckerman et al. 2003), as seems likely to have occurred for GD 362 (Becklin et al. 2005; Kilic et al. 2005; Jura et al. 2007) and G29-38 (Jura 2003; Reach et al. 2005a). While the disrupted minor-body model is promising, despite extensive surveys, only a handful of white dwarfs are known to display an infrared excess (Kilic et al. 2006a; Kilic \& Redfield 2007; Mullally et al. 2007; Farihi et al. 2007). We have therefore obtained Spitzer Space Telescope data to investigate the frequency of dusty disks orbiting white dwarfs and to assess models for the sources of the external pollution.

\section{OBSERVATIONS}

We observed 11 stars with atmospheric metals found in the SPY (SNe Ia Progenitor Survey) project (Koester et al. 2005) that also show a hint of $K$-band excess in Two Micron All Sky Survey (2MASS) photometry. We used the Infrared Array Camera (IRAC; Fazio et al. 2004) and Multiband Imaging Photometer for Spitzer (MIPS; Rieke et al. 2004) cameras on the Spitzer Space Telescope (Werner et al. 2004) to obtain photometry at wavelengths between 3.6 and $24 \mu \mathrm{m}$. IRAC observations were executed using a 20-point cycling dither pattern of medium step size, with $30 \mathrm{~s}$ individual exposures, yielding a total integration time of $600 \mathrm{~s}$ at all wavelengths. MIPS observations were performed using 10 cycles of the default 14-point dither pattern, with $10 \mathrm{~s}$ individual exposures, yielding a total integration time of $1400 \mathrm{~s}$. The data were processed with the IRAC and MIPS calibration pipelines (both ver. 14) to create single, reduced images on which to make photometric measurements. We removed

\footnotetext{
${ }^{1}$ Department of Physics and Astronomy and Center for Astrobiology, University of California, Los Angeles, CA 90095-1562; jura@astro.ucla.edu, ben@astro.ucla.edu.

${ }^{2}$ Gemini Observatory, Hilo, HI 96720; jfarihi@gemini.edu.
}

the one-dimensional artificial gradient in the sky, which is a common feature of MIPS pipelined data by subtracting a median collapsed image across the direction of the gradient. Aperture photometry was carried out with standard Image Reduction and Analysis Facility (IRAF) tasks, and measured fluxes were corrected for aperture size, but not for color. For IRAC detections, fluxes were typically measured in a 3 pixel radius aperture, but a 2 pixel radius aperture was used for faint sources or objects in a crowded field, and a 5 pixel radius aperture was used for GD 56, the brightest source. MIPS $24 \mu \mathrm{m}$ flux was measured in a 2.45 pixel radius aperture for GD 56, but a smaller aperture with a radius of 1.22 pixels was necessary for GD 133 because of a nearby galaxy. The measured fluxes were corrected to the standard IRAC and MIPS photometric apertures using corrections found in the most recent versions of the IRAC and MIPS data handbooks. The results are listed in Table 1. IRAC data were not obtained for two sources, since they are in other Spitzer programs; these data have not yet been published.

For both the IRAC and MIPS data, the photometric errors were estimated by taking the per pixel standard deviation in the extracted sky level and multiplying by the area of an aperture with a radius of 2 pixels. Color corrections have been ignored and are typically less than $1 \%$. Our dither pattern should remove the pixel phase dependent correction as well as the IRAC array location dependent errors described by Reach et al. (2005b). Total $1 \sigma$ errors (5\% calibration plus photometric measurement errors) are listed in Table 1. Because there is a somewhat larger scatter in the ratios of even the best-measured photospheric fluxes in the IRAC bands for white dwarfs (Farihi et al. 2007) than expected from model atmospheres (Tremblay \& Bergeron 2007; P. Bergeron 2007, private communication), to be conservative, following Hines et al. (2006) and Silverstone et al. (2006), we adopt a $10 \%$ calibration uncertainty in the reported fluxes, as reflected in the error bars displayed in Figures 1-4. Upper limits for nondetections in both IRAC and MIPS images were derived identically to the photometric errors for detections.

To assess the reliability of our IRAC photometry obtained with small apertures, we also reduced our data with CCDCAP. ${ }^{3}$ Although there is no significant difference with apertures of 3 pixel radii, it was found that IRAF reported fluxes 3\% smaller than CCDCAP with 2 pixel radius apertures at the two shorter wavelength IRAC channels where undersampling of the array

\footnotetext{
${ }^{3}$ Described at http://www.noao.edu/noao/staff/mighell/ccdcap.
} 
TABLE 1

Measured Infrared Fluxes

\begin{tabular}{|c|c|c|c|c|c|c|}
\hline Star & Other Name & $\begin{array}{c}F_{\nu}(3.6 \mu \mathrm{m}) \\
(\mu \mathrm{Jy})\end{array}$ & $\begin{array}{c}F_{\nu}(4.5 \mu \mathrm{m}) \\
\quad(\mu \mathrm{Jy})\end{array}$ & $\begin{array}{c}F_{\nu}(5.7 \mu \mathrm{m}) \\
(\mu \mathrm{Jy})\end{array}$ & $\begin{array}{c}F_{\nu}(7.9 \mu \mathrm{m}) \\
\quad(\mu \mathrm{Jy})\end{array}$ & $\begin{array}{c}F_{\nu}(24 \mu \mathrm{m}) \\
(\mu \mathrm{Jy})\end{array}$ \\
\hline WD $0300-013^{\mathrm{a}} \ldots \ldots \ldots \ldots \ldots \ldots$ & GD 40 & $231(12)$ & 199(10) & $159(15)$ & 164(17) & $<90$ \\
\hline WD $0408-041$ & GD 56 & $1090(55)$ & $1212(61)$ & $1177(61)$ & $1112(59)$ & $240(80)$ \\
\hline \multirow{2}{*}{ WD $1015+161 \ldots \ldots \ldots \ldots \ldots \ldots \ldots \ldots$} & PG $1015+161$ & 212(11) & $177(9)$ & $145(17)$ & $126(19)$ & $<110$ \\
\hline & PG $1015+161^{b}$ & $197(11)$ & $169(9)$ & $140(17)$ & $123(19)$ & $<110$ \\
\hline WD $1116+026$ & GD 133 & $592(30)$ & $527(26)$ & $472(27)$ & 464(29) & $310(100)$ \\
\hline WD $1124-293 \ldots \ldots \ldots \ldots \ldots \ldots \ldots$ & ESO $439-80$ & $\ldots$ & $\ldots$ & $\ldots$ & $\ldots$ & $<50$ \\
\hline 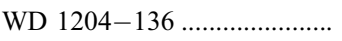 & EC $12043-1337$ & $158(5)$ & $99(4)$ & $72(14)$ & $32(17)$ & $<70$ \\
\hline WD $1225+006 \ldots \ldots \ldots \ldots \ldots \ldots$ & HE $1225+0038$ & $361(18)$ & $223(12)$ & $144(16)$ & $79(20)$ & $<80$ \\
\hline 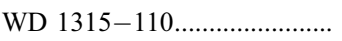 & HE $1315-1105$ & 210(11) & $131(7)$ & $90(15)$ & $30(18)$ & $<80$ \\
\hline 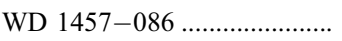 & PG $1457-086$ & $\ldots$ & $\ldots$ & $\ldots$ & $\ldots$ & $<90$ \\
\hline WD $2144-079^{\mathrm{a}} \ldots \ldots \ldots \ldots \ldots \ldots \ldots$ & G26-31 & 191(10) & $122(7)$ & $65(19)$ & $42(22)$ & $<100$ \\
\hline WD $2354+159^{\mathrm{a}} \ldots \ldots \ldots \ldots \ldots \ldots \ldots$ & PG $2354+159$ & $73(5)$ & $44(4)$ & $27(17)$ & $<21$ & $<110$ \\
\hline
\end{tabular}

NotE.-The $1 \sigma$ errors for detections are given in parentheses; the procedure for deriving the upper limits is described in $\S 2$.

${ }^{a}$ He-rich star (Koester et al. 2005).

b PG 1015+161 fluxes corrected for the background star, as discussed in $\S 2$.

is most significant. We have found that this small offset is largely mitigated by the aperture corrections provided by the Spitzer Science Center, and the net uncertainty introduced by using a small aperture is well below our $1 \sigma$ errors. As a further check, using 2 radius pixel aperture photometry and IRAF, we have reproduced the IRAC fluxes reported by Reach et al. (2005a) for G29-38 to within $3 \%$ at $4.5 \mu \mathrm{m}$ and to within $1 \%$ at $7.9 \mu \mathrm{m}$. We used 2 pixel radius apertures to reduce the data at 3.6 and $4.5 \mu \mathrm{m}$ for only one star, PG $2354+159$.

The data for PG $1015+161$ are contaminated by a star $\approx 2^{\prime \prime}$ away (Kilic et al. 2006a). Although our IRAC images are slightly elongated by $0.5^{\prime \prime}$ or 0.4 pixels at 3.6 and $4.5 \mu \mathrm{m}$ in the direction of this background star, we cannot accurately deconvolve the image, and we adopt an alternative approach to estimate its

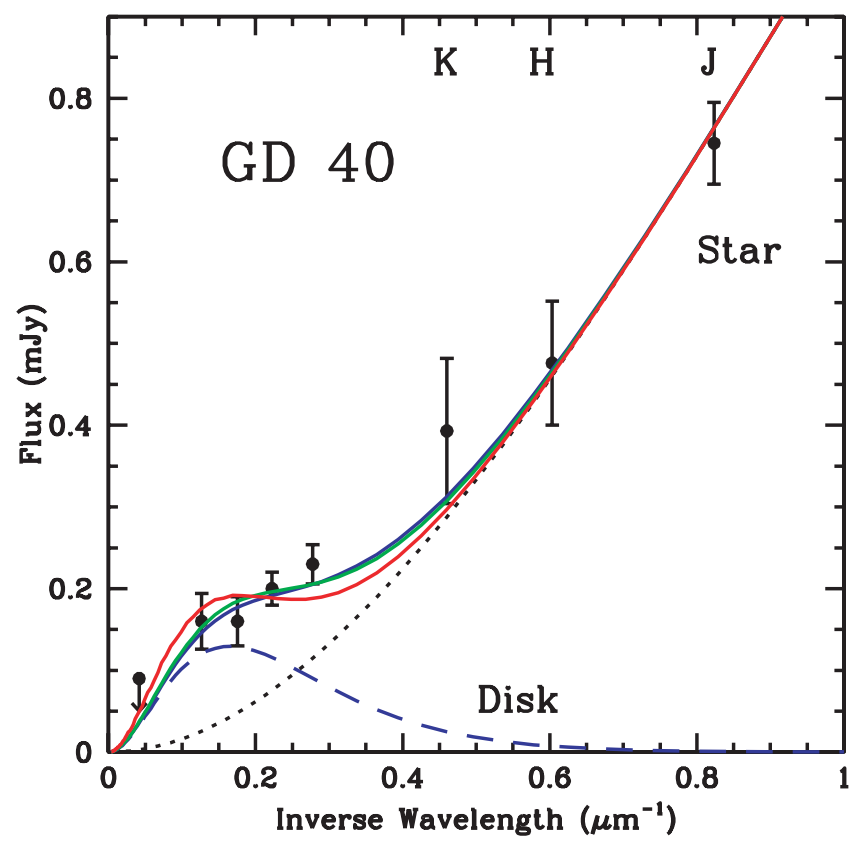

FIG. 1.-Comparison of models from Table 2 and data ( $2 \sigma$ errors) for GD 40. The plot shows the contribution from the stellar photosphere (dotted line), a disk model ( purple dashed line, model Agd40), and totals (solid purple line, model Agd40; solid green line, model Bgd40; solid red line, model Cgd40). Models Agd40 and Bgd40 are essentially indistinguishable, while model Cgd40 predicts somewhat too little flux at $3.6 \mu$ m because the warmest grains are only $800 \mathrm{~K}$. fluxes. As shown in Figure 5, a 1995.9 epoch Keck Observatory Near Infrared Camera (NIRC) $z$-band image (see Farihi et al. 2005), the angular separation of the background star was appreciably greater in the past, $2.9^{\prime \prime}$ versus $1.8^{\prime \prime}$ in 2007.0. From the Keck data, we find values of $z=19.12, J=18.76$, and $K=$ $18.26 \mathrm{mag}$, and infer a spectral type of approximately $\mathrm{K} 0 \mathrm{~V}$. We extrapolate these measures to IRAC wavelengths, and we list in Table 1 our results for PG 1015+161 after the estimated contributions from the background star are subtracted from the total measured IRAC fluxes at the position of PG 1015+161.

Both GD 133 and PG 1015+161 were suspected by Kilic et al. (2006a) to have an infrared excess on the basis of their $2 \mu \mathrm{m}$ spectra; our IRAC data demonstrate that these two stars indeed

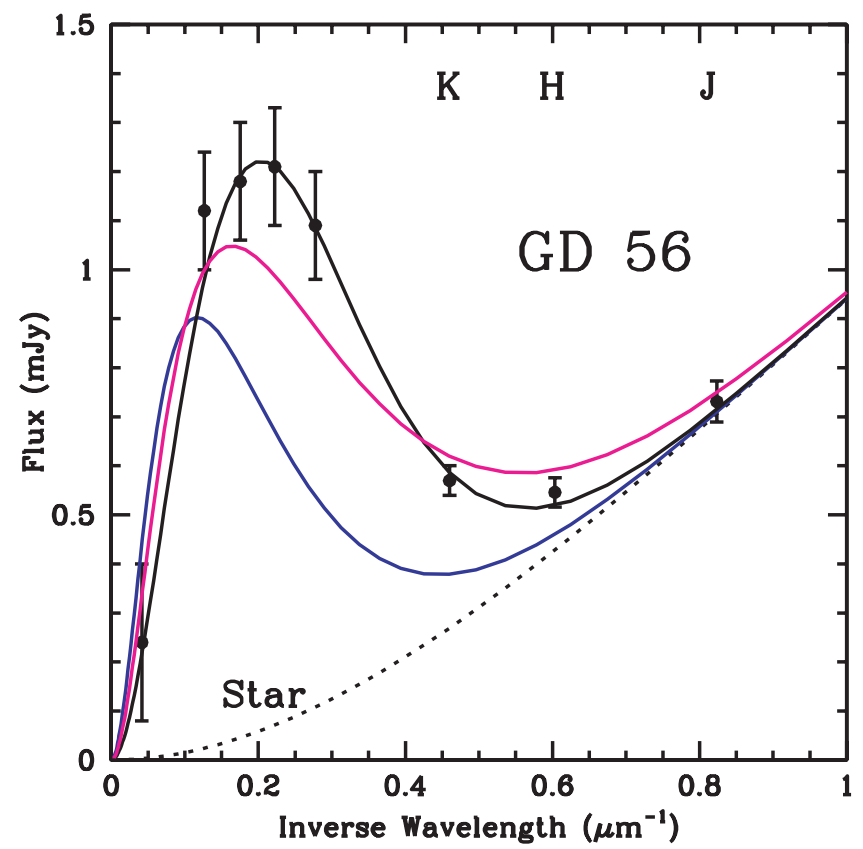

FIG. 2.-Comparison of three models and data ( $2 \sigma$ errors) for GD 56. The dotted line is the contribution from the stellar photosphere. The sums of the circumstellar and stellar fluxes are shown for models Agd56 (solid purple line) and Bgd56 (solid magenta line) listed in Table 2. We also show (solid black line) the flux for a model summing both the photosphere and excess emission for an opaque dust cloud at a temperature of $1000 \mathrm{~K}$ with an angular radius $1.4 \times 10^{-10} \mathrm{rad}$ as seen from Earth. 


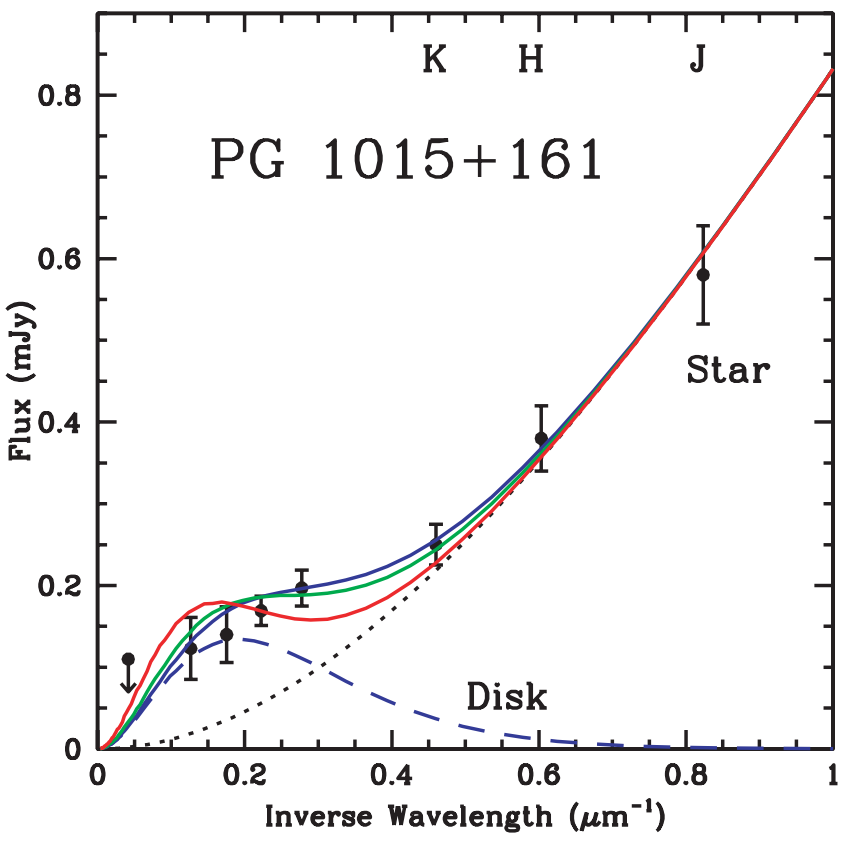

FIG. 3.- Similar to Fig. 1, but for PG 1015+161 with purple, green, and red for the totals of models Apg10, Bpg10, and $\mathrm{Cpg} 10$, respectively. The emission from only the disk is shown for model Apg10.

have excesses. GD 56 was previously shown to have a $2 \mu \mathrm{m}$ excess (Kilic et al. 2006a); our data significantly extend the wavelength range over which the excess is measured. Except for the slight hint in the 2MASS photometry, there is no previous measurement of an infrared excess for GD 40.

\section{MODELS}

We first try to account for the infrared data with the passive, opaque, flat disk model of Jura (2003). Here, the disk's potential vertical stratification is ignored, so that at each distance from the

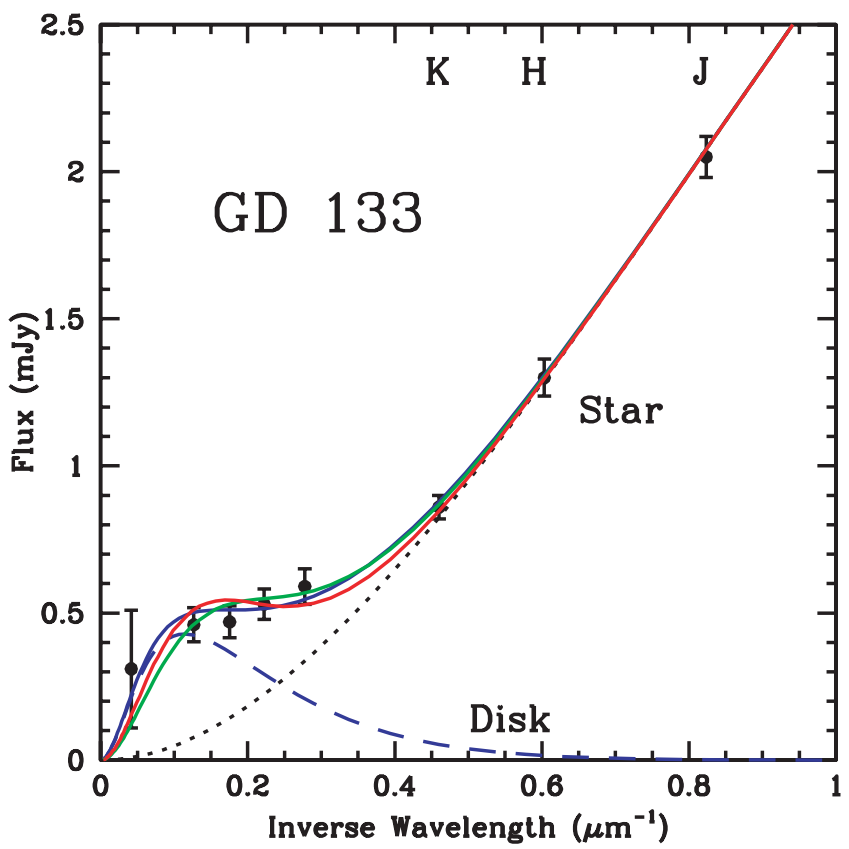

FIG. 4.- Similar to Fig. 1, but for GD 133 with purple, green, and red for the totals of models Agd133, Bpg13s, and Cpg133, respectively. The emission from only the disk is shown for model Agd133.

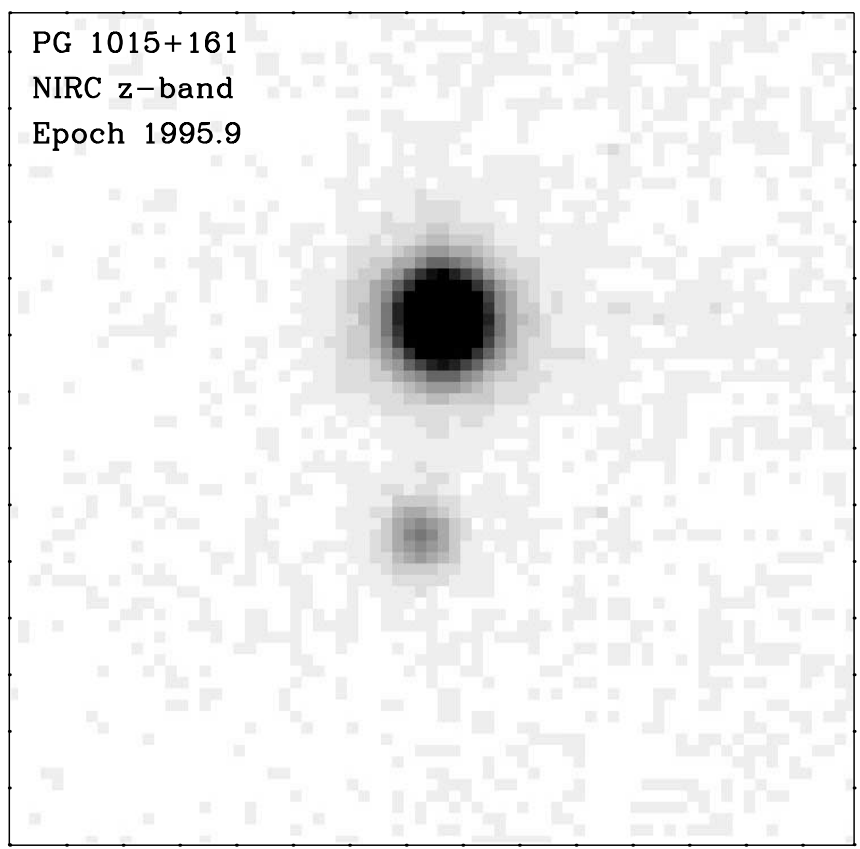

FIG. 5.-KECK NIRC $z$-band image, epoch 1995.9, obtained of PG 1015+ 161. The top of the image lies at P.A. $=96^{\circ}$; the background star lies $2.9^{\prime \prime}$ from the white dwarf at P.A. $=270^{\circ}$. The proper motion of $\mu=0.13^{\prime \prime} \mathrm{yr}^{-1}$ at P.A. $=239^{\circ}$ (Farihi et al. 2005) of PG $1015+161$ is consistent with its position relative to the background source in our 2007.0 IRAC images.

central star all dust grains achieve the same temperature as determined by the balance between radiative heating and cooling. Although a more sophisticated treatment is required to account for data such as the strong silicate feature seen in both the infrared spectrum of G29-38 (Reach et al. 2005a) and GD 362 (Jura et al. 2007), this simple model accounts for the currently available data.

Since all the target stars are warmer than $7000 \mathrm{~K}$, the received photospheric infrared flux from the star, $F_{\nu}(*)$, is modeled to better than the $2 \sigma$ measurement uncertainty as a singletemperature blackbody (Kilic et al. 2006b; Tremblay \& Bergeron 2007), $B_{\nu}$, so that

$$
F_{\nu}(*)=\frac{\pi R_{*}^{2}}{D^{2}} B_{\nu}\left(T_{*}\right),
$$

where the star of radius, $R_{*}$, and effective temperature, $T_{*}$, lie at distance $D$ from the Sun. For a flat, opaque disk that is passively illuminated by the host star, the expected flux, $F_{\nu}(d)$, is (Jura 2003)

$$
F_{\nu}(d)=12 \pi^{1 / 3} \frac{R_{*}^{2} \cos i}{D^{2}}\left(\frac{2 k_{\mathrm{B}} T_{*}}{3 h \nu}\right)^{8 / 3} \frac{h \nu^{3}}{c^{2}} \int_{x_{\min }}^{x_{\max }} \frac{x^{5 / 3}}{e^{x}-1} d x,
$$

where $i$ denotes the inclination angle of the disk and $i=0^{\circ}$ corresponds to a face-on configuration. Here, $x=(h \nu) /\left(k_{\mathrm{B}} T_{\text {disk }}\right)$, where $T_{\text {disk }}$ denotes the temperature in the disk. Since the disk is opaque, we cannot estimate its mass.

Table 2 lists the stellar parameters adopted from Friedrich et al. (2004), Koester et al. (2005), and Koester \& Wilken (2006), as well as our estimated disk parameters. The fits to our Spitzer data and near-infrared photometry from 2MASS or, when available, from the more accurate observations obtained at the Infrared Telescope Facility by Kilic et al. (2006a), are shown in 
TABLE 2

Stellar and Disk Properties

\begin{tabular}{|c|c|c|c|c|c|c|c|c|c|}
\hline Star & $\begin{array}{c}T_{*} \\
(\mathrm{~K})\end{array}$ & $\begin{array}{c}R_{*} \\
\left(R_{\odot}\right)\end{array}$ & $\begin{array}{c}R_{*} / D \\
\left(10^{-12}\right)\end{array}$ & Model & $\cos i$ & $\begin{array}{l}T_{\max } \\
(\mathrm{K})\end{array}$ & $\begin{array}{l}T_{\min } \\
(\mathrm{K})\end{array}$ & $\begin{array}{l}R_{\min } \\
\left(R_{*}\right)\end{array}$ & $\begin{array}{l}R_{\max } \\
\left(R_{*}\right)\end{array}$ \\
\hline \multirow[t]{3}{*}{ GD 40} & \multirow[t]{3}{*}{$15,200^{\mathrm{a}}$} & \multirow[t]{3}{*}{$0.013^{\mathrm{b}}$} & \multirow[t]{3}{*}{3.6} & $\operatorname{Agd} 40$ & 0.2 & 1200 & 600 & 18 & 44 \\
\hline & & & & $\operatorname{Bgd} 40$ & 0.4 & 1000 & 700 & 22 & 36 \\
\hline & & & & $\operatorname{Cgd} 40$ & 0.8 & 800 & 650 & 30 & 40 \\
\hline \multirow[t]{2}{*}{ GD 56} & \multirow[t]{2}{*}{$14,400^{\mathrm{a}}$} & \multirow[t]{2}{*}{$0.015^{\mathrm{c}}$} & \multirow[t]{2}{*}{3.6} & $\operatorname{Agd} 56$ & 1.0 & 1200 & 300 & 16 & 104 \\
\hline & & & & $\operatorname{Bgd} 56$ & 1.0 & 1700 & 400 & 10 & 71 \\
\hline \multirow[t]{3}{*}{ PG $1015+161 \ldots \ldots \ldots \ldots \ldots$} & \multirow[t]{3}{*}{$19,300^{\mathrm{a}}$} & \multirow[t]{3}{*}{$0.014^{\mathrm{c}}$} & \multirow[t]{3}{*}{2.7} & Apg10 & 0.3 & 1200 & 800 & 24 & 42 \\
\hline & & & & Bpg10 & 0.6 & 1000 & 800 & 31 & 42 \\
\hline & & & & Cpg10 & 1.0 & 800 & 700 & 42 & 50 \\
\hline \multirow{3}{*}{ GD 133} & \multirow{3}{*}{$12,200^{\mathrm{a}}$} & \multirow{3}{*}{$0.014^{\mathrm{c}}$} & \multirow[t]{3}{*}{7.0} & Agd133 & 0.2 & 1200 & 300 & 13 & 83 \\
\hline & & & & Bgd133 & 0.4 & 1000 & 600 & 17 & 33 \\
\hline & & & & Cgd133 & 0.8 & 800 & 600 & 23 & 33 \\
\hline
\end{tabular}

${ }^{a}$ Koester et al. (2005).

${ }^{b}$ Friedrich et al. (2004).

${ }^{\mathrm{c}}$ Derived from Koester \& Wilken (2006).

Figures 1-4. We find satisfactory fits for GD 40, GD 133, and PG $1015+161$ with a range of possible model parameters that are listed in Table 2. The inner dust temperature is constrained by the excess at $3.6 \mu \mathrm{m}$, but the fit is not unique. To fit the 4.5 and $5.7 \mu \mathrm{m}$ fluxes, there can be either a relatively broad range of temperatures with a more edge-on disk, or a narrower range of temperatures with a more face-on disk. In order not to overpredict the fluxes at 8 and $24 \mu \mathrm{m}$, the outer temperature cannot be too low. As listed in Table 2, using the thermal profile given in Jura (2003), the models lie within the tidal radius, which typically is about a factor of 100 greater than the star's radius (Davidsson 1999).

We see in Figure 1 that GD 40 can be equally well fit with a disk with an inner temperature of $1200 \mathrm{~K}$ that is nearly edge-on or one with an inner temperature of $1000 \mathrm{~K}$ that is more nearly face-on. If, however, the inner temperature is only $800 \mathrm{~K}$, then we cannot match the flux at $3.6 \mu \mathrm{m}$. Figure 3 displays the results for PG 1015+161. Again, there is little difference between a model that is more nearly edge-on with an inner temperature of $1200 \mathrm{~K}$ and a model that is more nearly face-on with an inner temperature near $1000 \mathrm{~K}$. However, a disk with an inner temperature of $800 \mathrm{~K}$ fails to produce enough flux at $3.6 \mu \mathrm{m}$. For GD 133, as shown in Figure 4, a model with an inner temperature of $1200 \mathrm{~K}$ that is viewed nearly edge-on is only somewhat better than a model with an inner temperature of $800 \mathrm{~K}$ that is viewed nearly face-on.

Kilic et al. (2006a) have shown that GD 56 is unusual in having a particularly marked excess at $2 \mu \mathrm{m}$, and as seen in Figure 2, our model Agd56 with an inner disk temperature of $1200 \mathrm{~K} \mathrm{com-}$ pletely fails. An alternative possibility with a less than satisfactory fit is that the inner disk temperature is larger than $1200 \mathrm{~K}$, and we also show in Figure 2 the results for model Bgd56 with an inner disk temperature of $1700 \mathrm{~K}$ and an outer disk temperature of $400 \mathrm{~K}$ (see Table 2). A better fit can be achieved if, rather than a flat disk, we assume that the disk is substantially warped as driven by the central star's luminosity (Pringle 1996) or otherwise puffed up by the gravitational field of a planet. To model this scenario, we assume a single-temperature blackbody at $1000 \mathrm{~K}$, and, as shown in Figure 2, we can fit the data if this material is a disk with an angular radius of $1.4 \times 10^{-10} \mathrm{rad}$ as seen from the Earth.

Our data are inconsistent with a simple model of interstellar accretion to explain the atmospheric metals. Assume interstellar grains are accreted at rate $\dot{M}_{\text {dust }}$ at the Bondi-Hoyle radius, $R_{\text {init }}$, which is typically between 1 and $10 \mathrm{AU}$ (Koester \& Wilken 2006), and then drift inward because of Poynting-Robertson drag to a final radius, $R_{\text {final }}$, where they are destroyed. In this scenario, the expected (see Jura 2006) infrared flux from interstellar (IS) accretion, $F_{\nu}($ IS), is

$$
F_{\nu}(\text { IS }) \approx\left[\frac{1}{2} \ln \left(\frac{R_{\text {init }}}{R_{\text {final }}}\right) \frac{\dot{M}_{\text {dust }} c^{2}}{\nu}\right] /\left(4 \pi D^{2}\right)
$$

This expression is valid for observations at frequencies where $h \nu / k$ lies between the minimum and maximum grain temperature. To evaluate equation (3), we adopt dust accretion rates that are 0.01 of the rates of accretion of interstellar gas given by Koester \& Wilken (2006) and also use their estimated distances. We adopt this value of the interstellar dust-to-gas ratio by mass of 0.01 from Zubko et al. (2004). For simplicity, we assume grains are destroyed by sublimation between $\sim 10^{-2}$ and $\sim 10^{-3} \mathrm{AU}$, the region where an unshielded grain that acts like a blackbody attains a temperature near $1200 \mathrm{~K}$. We therefore adopt $R_{\text {init }}=$ $1000 R_{\text {final }}$, although the results are insensitive to the exact value of this ratio. We show in Table 3 the predicted values of $F_{\nu}(24 \mu \mathrm{m})$ and a comparison between the predicted and observed fluxes, $F_{\text {pred }} / F_{\text {obs }}$, for the hydrogen-rich stars where the dwell time of metals in the atmosphere is typically less than $100 \mathrm{yr}$ (Koester $\&$ Wilken 2006), and therefore accretion is almost certainly

TABLE 3

Predicted $F_{\nu}(24 \mu \mathrm{m})$ For Simple Interstellar Accretion Followed by Poynting-Robertson Drag

\begin{tabular}{|c|c|c|c|c|}
\hline Star & $\begin{array}{c}D \\
(\mathrm{pc})\end{array}$ & $\begin{array}{c}d M_{\text {dust }} / d t \\
\left(10^{9} \mathrm{~g} \mathrm{~s}^{-1}\right)\end{array}$ & $\begin{array}{c}F_{\nu}(24 \mu \mathrm{m}) \\
\quad(\mathrm{mJy})\end{array}$ & $F_{\text {pred }} / F_{\text {obs }}$ \\
\hline . & 74 & 0.26 & 9.9 & 41 \\
\hline PG $1015+161 \ldots \ldots \ldots \ldots \ldots \ldots$ & 95 & 2.0 & 46 & $>420$ \\
\hline GD 133 & 43 & 0.37 & 42 & 140 \\
\hline ESO $439-80 \ldots \ldots \ldots$ & 34 & 0.13 & 23 & $>460$ \\
\hline EC $12043-1337 \ldots \ldots \ldots \ldots \ldots$. & 52 & 0.23 & 18 & $>260$ \\
\hline HE $1225+0038 \ldots \ldots \ldots \ldots \ldots$ & 29 & 0.0059 & 1.5 & $>19$ \\
\hline HE $1315-1105 \ldots \ldots \ldots \ldots \ldots$ & 40 & 0.022 & 2.9 & $>36$ \\
\hline PG 1457-086 & 117 & 1.5 & 23 & $>260$ \\
\hline
\end{tabular}

NotE.—Distances are taken from Koester \& Wilken (2006), while the dust accretion rates are scaled from their values, as described in $\S 3$. 
ongoing. In contrast, for the three helium-rich stars in our sample (GD 40, G26-31, and PG 2354+159), the atmospheric dwell times for metals are closer to $3 \times 10^{5} \mathrm{yr}$ (Paquette et al. 1986), and it is possible that accretion has stopped, but there are still metals lingering in the stellar photosphere. We see from Table 3 that the expected flux is always at least an order of magnitude larger than observed, and thus this simple model of interstellar accretion is unsatisfactory.

\section{DISCUSSION}

We find that four of nine white dwarfs with fluxes measured in the IRAC bands display evidence of circumstellar dust disks. For GD 40, the deficiency of carbon in the accreted material (Wolff et al. 2002) is naturally understood if an asteroid of at least $10^{23} \mathrm{~g}$ with a chondritic composition was tidally destroyed (Jura 2006), in agreement with some previous qualitative suggestions (Sion et al. 1990; Aannestad et al. 1993).

G29-38 was the first white dwarf found to have an infrared excess (Zuckerman \& Becklin 1987) from dust, while GD 362 was the second (Becklin et al. 2005; Kilic et al. 2005). With the results reported here and other recent studies (Mullally et al. 2007; Kilic et al. 2006a; Kilic \& Redfield 2007; Farihi et al. 2007), there are now enough white dwarfs with infrared excesses that it is possible to begin to discern some patterns. First, all the white dwarfs with an infrared excess also display atmospheric metals; thus, it is highly plausible that the stars are accreting from reservoirs of circumstellar material. Second, Kilic et al. (2006a), Farihi et al. (2007), and Kilic \& Redfield (2007) have shown that the stars with relatively high calcium abundances also tend to display an infrared excess. Third, the stars with an infrared excess all have effective temperatures greater than $\sim 9500 \mathrm{~K}$ and white dwarf cooling ages less than $\sim 1 \mathrm{Gyr} .{ }^{4} \mathrm{Ac}-$ cording to the simulations of Debes \& Sigurdsson (2002), most of the orbital perturbations of asteroids would occur during the first several hundred million years of the white dwarf's cooling, consistent with the data.

The scenario of a tidally disrupted asteroid explains the data for white dwarfs with both a relatively high photospheric calcium abundance and an infrared excess. However, the source of the atmospheric metals of those white dwarfs without an infrared excess is uncertain. One possibility is that the particle density is sufficiently low that mutual collisions lead to effective dust destruction. At, for example, the typical tidal radius of a white dwarf of $\sim 0.01 \mathrm{AU}$, the orbital speed is near $300 \mathrm{~km} \mathrm{~s}^{-1}$, and even small deviations from circular orbits can lead to mutual collision speeds in excess of $10 \mathrm{~km} \mathrm{~s}^{-1}$, which result in grain destruction. For stars with an infrared excess, the disks may be so dense that the grains act more like a granular fluid and the mutual collision speeds are small. Thus, the externally polluted white dwarfs without an infrared excess may have gas disks, as has been found for SDSS J122859.93+104032.9 (Gaensicke et al. 2006).

White dwarfs with a relatively low accretion rate tend not to possess an infrared excess. Scaling the accretion rates of Koester \& Wilken (2006) by 0.01 as described above, Figure 6 presents a comparison of $\dot{M}_{\text {dust }}$ versus effective temperature

\footnotetext{
${ }^{4}$ GD 362 previously was thought to be hydrogen-rich and to have a relatively low luminosity and therefore a cooling age well in excess of 1 Gyr (Gianninas et al. 2004). However, the star is now known to be helium-rich and to have a much larger luminosity and correspondingly shorter cooling age (Zuckerman et al. 2007). G167-8 has an effective temperature of $7400 \mathrm{~K}$, but its infrared excess, if real, is from much cooler dust than that found in the systems described here (Farihi et al. 2007).
}

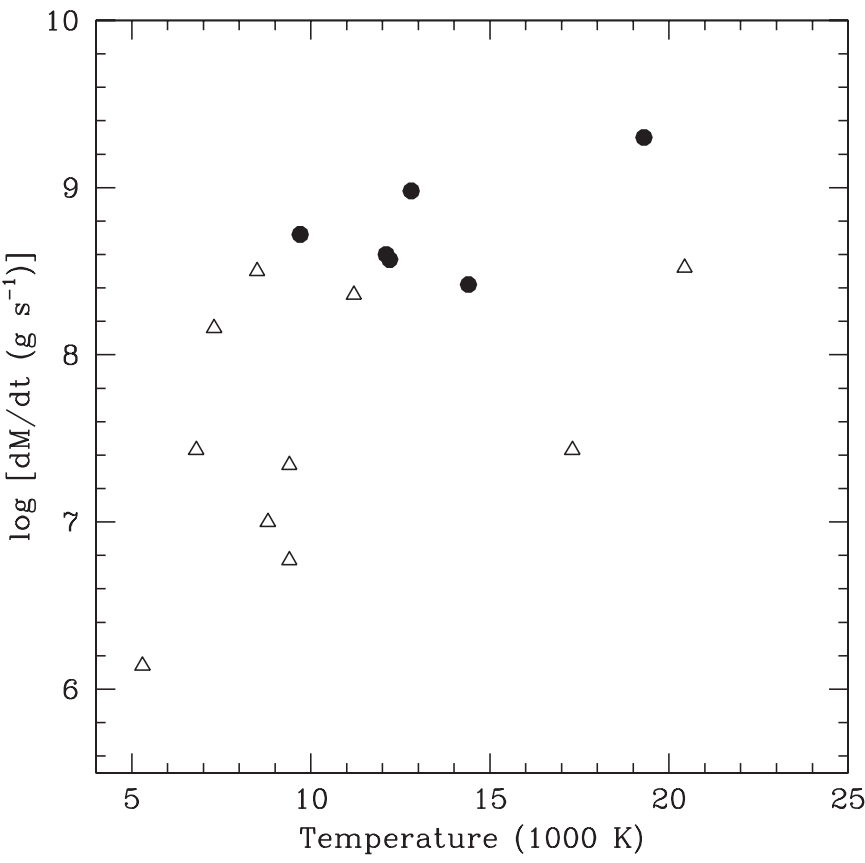

FIG. 6.-Mass accretion rates ( $(3)$ vs. effective temperature for hydrogenrich white dwarfs with an excess (circles) in either ground-based or Spitzer data, and without an excess (triangles) in published Spitzer data. GD 133 and PG $1015+161$ (this paper), GD 56 (this paper and Kilic et al. 2006a), G29-38 (Zuckerman \& Becklin 1987), WD 2115-560 (Mullally et al. 2007; von Hippel et al. 2007), and WD 1150-153 (Kilic \& Redfield 2007) have an excess, while EC 12043-1337, HE 1225+038, and HE 1315-1105 (this paper); WD 1202232, WD 1337+705, and WD 2149+021 (Mullally et al. 2007); and WD 0208+ 396, WD 0243-026, WD 0245+541, and WD 1257+278 (Debes \& Sigurdsson 2007) do not. Two helium-rich white dwarfs (GD 40 and GD 362) that also display an excess are not shown here.

for DAZs with IRAC photometry, distinguishing between those with and without excess emission. The correlation between having an infrared excess and $\dot{M}_{\text {dust }}$ shown in Figure 6 is closely related to the result found by Kilic et al. (2006a) that the stars with a greater metal abundance are the ones with an infrared excess. Although the numbers are very limited, the DAZs with $\dot{M}_{\text {dust }}$ larger than about $3 \times 10^{8} \mathrm{~g} \mathrm{~s}^{-1}$ possess an excess, while the stars with lower accretion rates do not. For comparison, the dust production rate in the zodiacal cloud is about $3 \times 10^{6} \mathrm{~g} \mathrm{~s}^{-1}$ (Fixsen \& Dwek 2002) and considerably larger around some other main-sequence stars. Even in their advanced evolutionary state, some white dwarfs may possess a population of eroding parent bodies.

\section{CONCLUSIONS}

We have found evidence for dusty disks orbiting four externally polluted white dwarfs. For GD 40, the evidence lends support to the hypothesis that tidal disruption of a carbon-deficient asteroid has occurred.

This work has been partly supported by NASA and is based on observations made with the Spitzer Space Telescope, which is operated by the Jet Propulsion Laboratory, California Institute of Technology, for NASA. We thank P. Bergeron for sending us models of white dwarf atmospheres, M. Kilic for useful correspondence, and T. von Hippel for a helpful referee's report. 


\section{REFERENCES}

Aannestad, P. A., Kenyon, S. J., Hammond, G. L., \& Sion, E. M. 1993, AJ, 105,1033

Becklin, E. E., Farihi, J., Jura, M., Song, I., Weinberger, A. J., \& Zuckerman, B. 2005, ApJ, 632, L119

Davidsson, B. J. R. 1999, Icarus, 142, 525

Debes, J. H., \& Sigurdsson, S. 2002, ApJ, 572, 556 2007, in ASP Conf. Ser. 372, 15th European White Dwarf Workshop ed. R. Napiwotzki \& M. Barstow (San Francisco: ASP), in press (astro-ph/ 0703448)

Farihi, J., Becklin, E. E., \& Zuckerman, B. 2005, ApJS, 161, 394

Farihi, J., Zuckerman, B., \& Becklin, E. E. 2007, ApJ, submitted

Fazio, G. G., et al. 2004, ApJS, 154, 10

Fixsen, D. J., \& Dwek, E. 2002, ApJ, 578, 1009

Friedrich, S., Jordan, S., \& Koester, D. 2004, A\&A, 424, 665

Gaensicke, B. T., Marsh, T. R., Southworth, J., \& Rebassa-Mansergas, A. 2006, Science, 314, 1908

Gianninas, A., Dufour, P., \& Bergeron, P. 2004, ApJ, 617, L57

Hines, D., et al. 2006, ApJ, 638, 1070

Jura, M. 2003, ApJ, 584, L91

$$
\text { 2006, ApJ, 653, } 613
$$

Jura, M., Farihi, J., Zuckerman, B., \& Becklin, E. E. 2007, AJ, 133, 1927

Kilic, M., \& Redfield, S. 2007, ApJ, 660, 641

Kilic, M., von Hippel, T., Leggett, S. K., \& Winget, D. E. 2005, ApJ, 632, L115 2006a, ApJ, 646, 474

Kilic, M., von Hippel, T., Mullally, F., Reach, W. T., Kuchner, M. J., Winget, D. E., \& Burrows, A. 2006b, ApJ, 642, 1051
Koester, D., Rollenhagen, K., Napiwotzki, R., Voss, B., Christlieb, N., Homeier, D., \& Reimers, D. 2005, A\&A, 432, 1025

Koester, D., \& Wilken, D. 2006, A\&A, 453, 1051

Mullally, F., Kilic, M., Reach, W. T., Kuchner, M. J., von Hippel, T., Burrows, A., \& Winget, D. E. 2007, ApJS, 171, in press (astro-ph/0611588)

Paquette, C., Pelletier, C., Fontaine, G., \& Michaud, G. 1986, ApJS, 61, 197 Pringle, J. E. 1996, MNRAS, 281, 357

Reach, W. T., Kuchner, M. J., von Hippel, T., Burrows, A., Mullally, F., Kilic, M., \& Winget, D. E. 2005a, ApJ, 635, L161

Reach, W. T., et al. 2005b, PASP, 117, 978

Rieke, G. H., et al. 2004, ApJS, 154, 25

Silverstone, M., et al. 2006, ApJ, 639, 1138

Sion, E., Hammond, G. L., Wagner, R. M., Starrfield, S. G., \& Liebert, J. 1990, ApJ, 362, 691

Tremblay, P. E., \& Bergeron, P. 2007, ApJS, in press

von Hippel, T., Kuchner, M. J., Kilic, M., Mullally, F., \& Reach, W. T. 2007, ApJ, 662, 544

Werner, M. W., et al. 2004, ApJS, 154, 1

Wolff, B., Koester, D., \& Liebert, J. 2002, A\&A, 385, 995

Zubko, V., Dwek, E., \& Arendt, R. G. 2004, ApJS, 152, 211

Zuckerman, B., \& Becklin, E. E. 1987, Nature, 330, 138

Zuckerman, B., Koester, D., Reid, I. N., \& Hunsch, M. 2003, ApJ, 596, 477

Zuckerman, B., et al. 2007, ApJ, submitted 